\title{
IONIZED AND NEUTRAL GAS IN THE CENTRAL 10 PC OF THE GALAXY
}

\author{
K. Y. LO, R. PLANTE \\ University of Illinois, Astronomy dept., Urbana, IL 61801, U. S. A. \\ J. LACY \\ University of Texas, Astronomy dept., Austin, TX 78712, U. S. A. \\ M. WRIGHT \\ University of California, Radio Astronomy Lab., Berkeley, CA 94720, \\ U. S. A. \\ N. KILLEEN \\ ATNF, P. O. Box 76, 2121 Epping, NSW, Australia
}

\begin{abstract}
Observations of the $12.8 \mu \mathrm{m}$ [NeII] line emission, the $\mathrm{OH}$ absorption against Sgr $\mathrm{A}$ and the HCN emission were compared to determine the relationship between the ionized and neutral gas in the central $10 \mathrm{pc}$ of the Galaxy. The distribution of the neutral gas is too asymmetric to be a ring or disk. Along the western arc, the ionized gas velocity is very different from that of the neutral gas, suggesting it may not be the ionized inner edge of the circum-nuclear neutral gas.
\end{abstract}

\section{INTRODUCTION}

The gas distribution within the central $10 \mathrm{pc}$ of the Galaxy is generally well described by a 2.5-pc diameter cavity surrounded by a ring-like distribution of highly clumpy high density molecular gas (and dust), with high velocity dispersion (Becklin et al 1982; Liszt et al 1983; Lo and Claussen 1983; Genzel et al 1985; Güsten et al 1987; Genzel 1989). The central cavity is filled with filamentary structure of ionized gas (Ekers et al 1983; Lo and Claussen 1983) and neutral atomic gas (Jackson et al 1993). It was proposed by Lo and Claussen (1983) that an explosion at the center $\sim 10^{4}$ years ago created the central cavity and that the ionized streamers represent tidally stretched molecular clouds falling in towards the center from the surrounding neutral material.

In projection, the spatial complementarity of the integrated $\mathrm{HCN}$ emission and radio continuum of Sgr A West is strikingly good (Güsten et al 1987). It does imply the circumnuclear neutral gas, as represented by the HCN emission, is physically related to the ionized gas, represented by the radio free-free emission. However, the 3-dimensional relationship between the ionized and neutral gas has not been analyzed in detail. For example, if the ionzed gas along the Western Arc is indeed the ionized inner edge of the neutral gas, the ionized gas should 
have similar kinematics as the adjacent neutral gas.

In order to study the spatial and kinematic relationship between the neutral and ionized gas within the central $10 \mathrm{pc}$, we have compared recent high resolution spectroscopic observations of the $12.8 \mu \mathrm{m}$ emission (1" resolution), VLA $\lambda 18 \mathrm{~cm}$ OH absorption against Sgr A (3" $\times 4 ")$, BIMA $\lambda 3.4 \mathrm{~mm} \mathrm{HCN}$ emission (4") and the VLA $\lambda 6 \mathrm{~cm}$ continuum map $\left(0.3 " \times 0.6\right.$ ") from $\mathrm{Sg}_{\mathrm{I}} \mathrm{A}$ West. Comparing the $\mathrm{OH}$ absorption against the $\mathrm{HCN}$ emission from the circumnuclear neutral gas may provide some information on the line-of-sight placement of the neutral gas relative to the continuum source Sgr A.

\section{THE DATA}

The $12.8 \mu \mathrm{m}$ [Ne II] data was obtained with the spectrometer at IRTF (cf. Lacy et al 1991) with resolutions of 1 " and $33 \mathrm{~km} / \mathrm{s}$. The $\mathrm{OH}$ absorption against Sgr A was originally obtained for the Zeeman measurements (Killeen, Lo and Crutcher 1992 ) and has a resolution of 3 " $\times 4$ " and $2 \mathrm{~km} / \mathrm{s}$. The BIMA HCN emission maps were derived from additional high resolution observations combined with those published by Giisten et al (1987). The radio continuum map is that published in Killeen and Lo (1989),

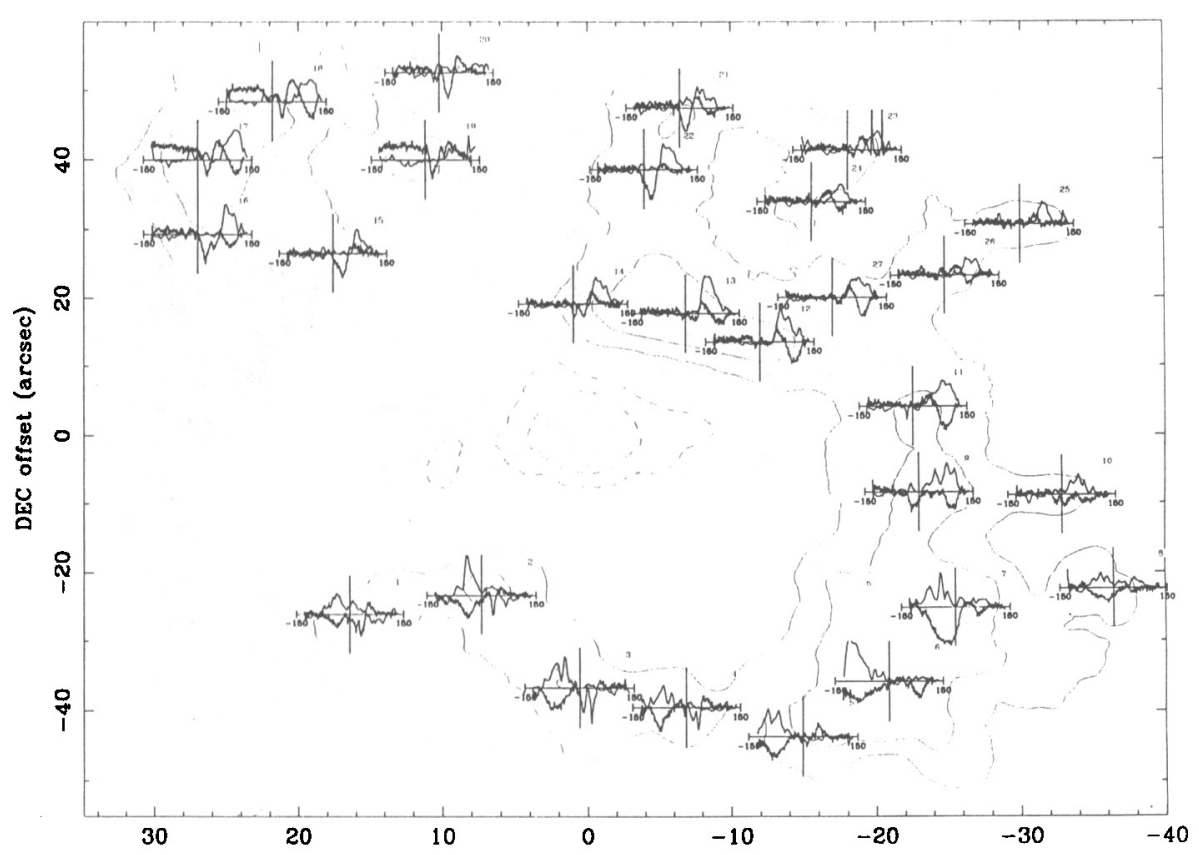

Figure 1: Comparison of $\mathrm{HCN}$ emission and $\mathrm{OH}$ absorption. The origin is at $\operatorname{Sgr} A^{*}$. 


\section{RESULTS}

\section{Sgr A and Circumnuclear Neutral Gas}

The $\lambda 18 \mathrm{~cm} \mathrm{OH}$ line absorption against $\mathrm{Sgr} \mathrm{A}$ (East and West) presumably arises from the same neutral gas responsible for the $\lambda 3.4 \mathrm{~mm}$ HCN emission. As indicated by the spectra plotted at different points of the circumnuclear neutral material (Figure 1), there is much similarity of the two tracers. Generally, whenever there is $\mathrm{HCN}$ emission (especially in the velocity range between -120 to $-20 \mathrm{~km} / \mathrm{s}$, and between 40 to $100 \mathrm{~km} / \mathrm{s}$ ), there is the corresponding $\mathrm{OH}$ absorption. However, in the quadrant to the north and to the west of the origin, the HCN emission 40 to $100 \mathrm{~km} / \mathrm{s}$ tends to have weaker $\mathrm{OH}$ absorption. Also, there exists $\mathrm{OH}$ absorption between -10 to $40 \mathrm{~km} / \mathrm{s}$ without much corresponding HCN emission, likely to be due to foreground gas of lower density.

\section{Ionized Gas and Neutral Gas}

Figure 2 shows a series of $\mathrm{HCN}$ and [NeII] emission spectra at varions locations along the Western Arc. While spectra 1 (RA offset $=-10 "$, DEC offset $=-40 "$ ) through 4 , and 12 through 16 (RA offset $=25 "$ " DEC offset $=20 "$ ) show very good correspondence between $\mathrm{HCN}$ and $[\mathrm{NeII}]$ line profiles, there are distinct

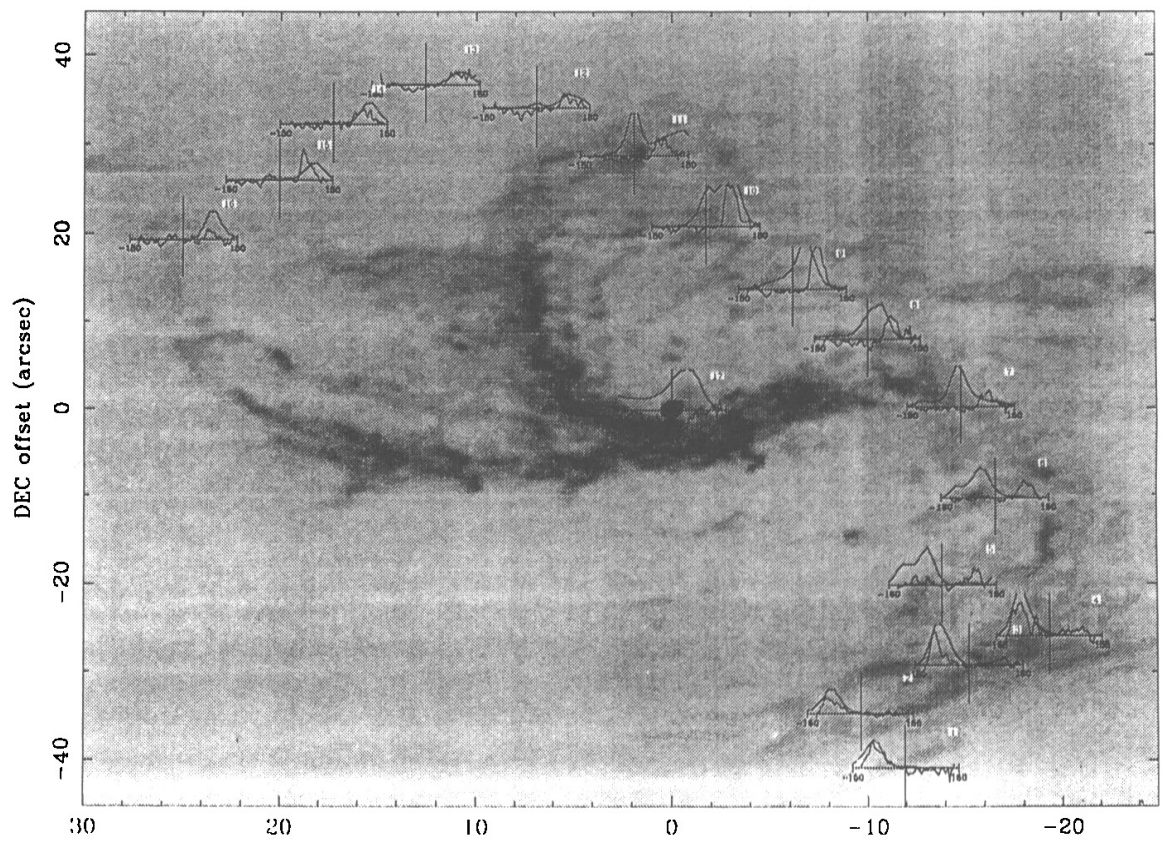

Figure 2: Comparison of $12.8 \mu \mathrm{m}$ [NeII] to $3.4 \mathrm{~mm} \mathrm{HCN}$ emission. The smoother profiles are $[\mathrm{NeII}]$ emission. The origin is at $\mathrm{Sgr} \mathrm{A*}$. 
differences in spectra 5 through 11 . In particular, while the [NeII] emission apparently shifts its velocity continuously from $-100 \mathrm{~km} / \mathrm{s}$ at DEC offset $=-40$ " to $100 \mathrm{~km} / \mathrm{s}$ at DEC offset $=40 "$, the HCN emission appears to shift abruptly from $-100 \mathrm{~km} / \mathrm{s}$ in spectrum 4 to $100 \mathrm{~km} / \mathrm{s}$ in spectrum 5 . This is not due to the absorption of HCN emission by foreground gas, since the foreground gas is not apparently in the $\mathrm{OH}$ absorption. Thus, the kinematics of [NeII] (ionized gas) and HCN (neutral gas) in the same location along the Western Arc is very different. This indicates that the ionized gas along the Western Arc cannot be the ionized inner edge of the circumnuclear neutral gas. It is consistent with the suggestion that parts of the neutral material have broken off the circumnuclear neutral material and are falling into the center, being tidally stretched and ionized in the process (Lo and Claussen 1983).

Furthermore, comparisons of the distribution of [NeII] emission, HCN emission and $\mathrm{OH}$ absorption at different corresponding red and blue shifts about 0 $\mathrm{km} / \mathrm{s}$ all show gross differences, indicating that the $11, \mathrm{tral}$ and ionized gas cannot be in a simple circumnuclear disk or ring.

\section{CONCLUSION}

The distribution of the circumnuclear neutral gas is very asymmetric, indicating the gas is unlikely to be in a disk or ring. A detailed comparison of the various tracers of the ionized and neutral gas will present a more complicated picture of the gas within the central $10 \mathrm{pc}$ and may help to delineate the origin of the observed cavity, the ionized filaments and the circumnuclear gas.

\section{REFERENCES}

Becklin, E. et al 1982, ApJ, 258, 135

Ekers, R. et al 1983, A\&A, 122, 143

Genzel, R. et al 1985, ApJ, 297, 766

Genzel, R. 1989, IAU Symposium 136, 393

Güsten, R. et al 1987, ApJ, 318, 124

Jackson, J. et al 1993, ApJ, 402, 173

Killeen, N., Lo, K. Y. 1989, IAU Symposium 136, 453

Killeen, N., et al 1992, ApJ, 385, 585

Lacy, J. et al 1991, ApJ, 380, L71

Liszt, H. et al 1983, $A \& A, \mathbf{1 2 6}, 341$

Lo, K. Y., Claussen, M. 1983, Nature, 306, 647 


\section{DISCUSSION}

M. Hayashi If we observe Sgr $\mathrm{A}^{*}$ at $8 \mathrm{GHz}$ through the scattering interstellar medium, how do you interpret the elongated structure observed with $8 \mathrm{GHz}$ VLBA experiments?

K. Y. Lo The principal source of interstellar electron scattering the radio emission from SgrA* appears to be due to those in the central few pc and may very well be affected by the fast $\left(1000-2000 \mathrm{~km} \mathrm{~s}^{-1}\right)$ winds from the recently discovered He line stars. It is possible that the properties of such a medium can be anisotropic.

H. Zinnecker Can you tell us more about your 1 micron HST observations of the Galactic Centre? How do they compare to the ground-based observations?

K.Y. Lo The $1 \mu \mathrm{m}$ HST Planetary Camera image of the Galactic center was made in July 1991 with average of 210 -min exposures. Because of the well-known problem of the HST, the resulting image does not add much to ground -based $1 \mu \mathrm{m}$ images. However, we (J. Biretta and Lo) have been awarded an additional 7 orbits of HST observing time which should be scheduled in mid-1993. We look forward to having a 0.1 "resolution WF camera image of the Galactic Center at $1 \mu \mathrm{m}$. 